\title{
Probe captures neuronal conversations in people
}

\author{
BY HOLLY BARKER
}

9 FEBRUARY 2022

\section{Listen to this story:}

https://www.spectrumnews.org/wp-

content/uploads/2022/02/audio-4f2b383c-61fe-4d7b-8709-200c8f22e4ff-encodings.mp3

A high-resolution probe commonly used in animals can be modified to simultaneously record the activity of hundreds of individual neurons across the human brain. The tool could be used to study neuronal activity underlying seizures, which sometimes accompany autism, its makers say.

Devices traditionally used in people can record the electrical activity of only a few dozen individual brain cells at a time. A popular, ultra-thin probe called Neuropixels - containing close to 1,000 electrodes spaced 20 micrometers apart - can detect signals from more than 200 individual neurons across large sections of the brain in mice and other small animals.

Originally designed for rodents, though, the probe has proved too fragile for use in larger brains, says Angelique Paulk, instructor in neurology at Massachusetts General Hospital in Boston. So she and her colleagues attached a thicker shank to the device.

The team used the modified, sterilized Neuropixels probes to take simultaneous measurements at 384 of the device's 960 recording sites in three people - all of whom were already due to undergo craniotomy, which exposes the brain, to treat a movement disorder or severe epilepsy.

The researchers had just 15 minutes during each surgery to make the recordings, and they used a robotic arm to guide the probe into the cerebral cortex with precision.

The modified probe detected electrical activity from hundreds of neurons across different layers of the cerebral cortex in the three participants. An algorithm processed the data to account for brain movement due to blood flow and breathing. Unlike the isolation of research labs, the hospital 


\section{Spectrum | Autism Research News}

https://www.spectrumnews.org

setting creates additional 'noise,' such as the sounds of hospital equipment and patients in adjacent rooms, which also needs to be factored into this processing step, Paulk says.

Open-source software that sorts patterns of neuronal activity into clusters enabled the team to track the location and identity of single cells across the recordings. The work appeared 31 January in Nature Neuroscience.

In a 2021 study, researchers implanted electrodes in the brains of people undergoing surgery and identified neurons involved in 'theory of mind,' or the ability to perceive the thoughts and intentions of others. Because Neuropixels probes are more sensitive, they might be used to reveal how these neurons interact with other brain regions, Paulk says.

\section{Cite this article: https://doi.org/10.53053/UXOZ9061}

